\title{
Volumetric characteristics of idiopathic pulmonary fibrosis lungs: computational analyses of high-resolution computed tomography images of lung lobes
}

\author{
Bora Sul ${ }^{1,2}$, Lucia Flors ${ }^{3}$, Joanne Cassani ${ }^{3}$, Michael J. Morris ${ }^{4}$, Jaques Reifman ${ }^{1 *}$ (D) Talissa Altes ${ }^{3}$ and
} Anders Wallqvist ${ }^{1}$

\begin{abstract}
Background: Idiopathic pulmonary fibrosis (IPF) is a fatal lung disease involving progressive degeneration of lung capacity. Current diagnosis of IPF heavily relies on visual evaluation of fibrotic features in high-resolution computed tomography (HRCT) images of the lungs. Although the characteristics of this disease have been studied at the molecular and cellular levels, little is known about the mechanical characteristics of IPF lungs inferred from HRCT images. To this end, we performed a pilot study to investigate the radiographic and volumetric characteristics of lungs in subjects with IPF.

Methods: We collected HRCT images of healthy $(N=13)$ and IPF $(N=9)$ lungs acquired at breath-holds after full inspiration (expanded state) and full expiration (contracted state). We performed statistical analyses on Hounsfield unit (HU) histograms, lobar volumes (V: lobe volume normalized by the lung volume), and lobar flows (Q: the difference in lobe volume divided by the difference in lung volume between the expanded and contracted states).

Results: Parameters characterizing the HU histograms (i.e., mean, median, skewness, and kurtosis) significantly differed between healthy and IPF subjects, for all lobes in both expanded and contracted states. The distribution of $V$ across lobes differed significantly between the groups in both states. The distribution of $Q$ also differed significantly between the groups: Q values of the lower lobes for the IPF group were 33\% (right) and 22\% (left) smaller than those for the healthy group, consistent with the observation that radiographic scores were highest in the lower lung section in IPF. Notably, the root-mean-squared difference (RMSD) of $Q$, a measure of distance from the mean value of the healthy group, clearly distinguished the IPF subjects (RMSD of $Q>1.59$ ) from the healthy group (RMSD of $Q<0.67$ ).

Conclusion: This study shows that lung volume and flow distribution change heterogeneously across the lung lobes of IPF subjects, with reduced capacity in the lower lobes. These volumetric changes may improve our understanding of the pathophysiology in IPF lungs.
\end{abstract}

Keywords: Idiopathic pulmonary fibrosis, Quantitative HRCT analysis, Lobar flow distribution

\footnotetext{
* Correspondence: jaques.reifman.civ@mail.mil

'Department of Defense Biotechnology High Performance Computing

Software Applications Institute, Telemedicine and Advanced Technology Research Center, United States Army Medical Research and Development Command, FCMR-TT, 504 Scott Street, Fort Detrick, MD 21702-5012, USA Full list of author information is available at the end of the article
}

(c) The Author(s). 2019 Open Access This article is distributed under the terms of the Creative Commons Attribution 4.0 International License (http://creativecommons.org/licenses/by/4.0/), which permits unrestricted use, distribution, and reproduction in any medium, provided you give appropriate credit to the original author(s) and the source, provide a link to the Creative Commons license, and indicate if changes were made. The Creative Commons Public Domain Dedication waiver (http://creativecommons.org/publicdomain/zero/1.0/) applies to the data made available in this article, unless otherwise stated. 


\section{Background}

Idiopathic pulmonary fibrosis (IPF) is a specific form of chronic and fibrosing interstitial pneumonia of unknown cause [1-3], and the most common and fatal among such interstitial lung disorders. Patients with IPF are characterized by progressively worsening dyspnea and have a median life expectancy of three to five years from the time of diagnosis [4].

Previous studies have identified molecular and cellular mechanisms [5-8] that are potentially associated with the onset and progression of IPF. More recently, a number of studies have demonstrated that the structural and mechanical characteristics of extracellular matrices and fibroblasts differ between normal and IPF tissues [9-11]. A particularly interesting study has shown that tissue stiffness is greater in fibroblasts from patients with IPF than those from normal subjects [12]. These studies suggest that changes in mechanical and structural characteristics of tissues could directly lead to reduced lung function in IPF subjects. Such changes could also affect lung function indirectly, as the behavior and structural characteristics of tissues could further change via positive feedback between stiffened tissues and their microenvironments $[13,14]$.

Several imaging studies, particularly those using high-resolution computed tomography (HRCT), have characterized abnormalities in the lungs of IPF patients $[2,15,16]$. These studies have shown that excessive and abnormal fibrosis in IPF lungs are manifested as numerous abnormal radiological features [17], including subpleural and basilar predominant reticulation, honeycombing, traction bronchiectasis and bronchiolectasis, and ground-glass opacification $[15,18]$. In particular, these features have been invaluable in establishing HRCT imaging as a critical tool in diagnosing $[15,16,19]$, staging, or monitoring the progression of IPF [20-22].

Despite these advances, diagnosis of IPF heavily relies on visual evaluation of the described fibrotic features in HRCT images, and assessment of the disease is subjective with no commonly accepted or standardized fibrotic score. In addition, we still have only a rudimentary understanding of how the structural changes in the lungs of IPF subjects affect their functional properties. Although increases in the stiffness of tissues are likely to reduce global lung function, whether such changes induce regional differences in the mechanical responses of lungs is not well understood. In addition, it is of interest to determine whether HRCT images can be used to detect such regional changes in lung function. To address these questions, here we investigated the radiological and volumetric characteristics of the lungs in healthy and IPF subjects. In particular, we compared mechanical quantities for individual lung lobes in both expanded and contracted lung states by quantitatively analyzing HRCT images.

\section{Methods \\ Study subjects and pulmonary function tests}

We recruited healthy and IPF subjects through advertisements in local hospitals near the University of Missouri, Columbia, MO. All IPF subjects enrolled in this study were diagnosed with the disease following the usual clinical work-up with imaging. None of the subjects had been diagnosed with emphysema at the time of enrollment. Although one subject (D9) showed mild signs of emphysema on imaging, this person had no clinical diagnosis of emphysema (e.g., $\mathrm{FEV}_{1}=97 \%$ predicted). No other subject was diagnosed with COPD or emphysema, although many had shortness of breath.

Initially, we selected the IPF subjects based on their previous medical history, including the initial diagnosis and most recent prognosis of IPF based on HRCT imaging obtained prior to enrollment. Clinical diagnosis of IPF was based on clinical course, pulmonary function tests, and HRCT findings for all patients. According to the guidelines of the 2018 ATS [15] and the Fleischner Society [23], the UIP patterns were typical for six patients (D1, D2, D3, D6, D7, and D9), probable for two (D4 and D5), and indeterminate for two (D8 and one excluded from the study. We excluded this patient because the large amount of fibrosis in the lung led to high uncertainty in the segmentation of lung lobes.) Both patients with indeterminate UIP patterns and one (D4) with probable had a lung biopsy as part of the initial clinical work-up. The remaining patients were diagnosed based on their original clinical work-up and CT findings. Given that our investigation was a pilot study focused on imaging, we did not perform any additional testing (e.g., autoimmune panel testing) to confirm the original diagnosis of IPF or exclude alternative diagnoses. Healthy subjects had no history of pulmonary disease and had smoked less than 100 cigarettes in their lifetime. One healthy subject (H13) had smoked a few cigarettes over a 6-month period (less than 100 over a lifetime). None of the other healthy subjects had ever smoked. Four IPF subjects (D5, D6, D8, and D9) had a history of smoking (past or current) ranging from 15 to 20 years. One subject (D1) had smoked more than 40 years ago, but less than 100 cigarettes over a lifetime. The remaining IPF subjects had never smoked. All subjects were more than 18 years of age.

For each subject, we performed a full pulmonary function test (PFT; Vmax Encore PFT system, Vyaire Medical, Mettawa, IL), including measurements for lung volumes and diffusing capacity of carbon monoxide (DLCO). For the functional tests, we provided the values for the forced vital capacity (FVC), the forced expiration 
volume during one second $\left(\mathrm{FEV}_{1}\right)$, the total lung capacity (TLC), and DLCO as \% predicted, i.e., as a percentage of the normal reference value provided by the National Health and Nutritional Examination Survey III [24] and European Respiratory Society [25]. We defined the $\mathrm{FEV}_{1} / \mathrm{FVC}$ quantity conventionally as the ratio of the raw volumetric measurements (in liters) of $\mathrm{FEV}_{1}$ and FVC rather than as a ratio of \%-predicted values.

For the physiology-based risk assessment of IPF, we calculated the gender-age-physiology (GAP) index [20], a composite score (range: $0-8$ ) that considers scores for gender $(\mathrm{G})$, age $(\mathrm{A})$, and physiology $(\mathrm{P})$. $\mathrm{G}$ is scored as 0 for women and 1 for men, and $\mathrm{A}$ as 0,1 , or 2 for age ranges $\leq 60,61-65$, or $>65$, respectively. $P$ denotes the sum of the subscores, $\mathrm{P}_{\mathrm{FVC}}$ and $\mathrm{P}_{\mathrm{DLCO}}$, which are associated with the FVC and DLCO, respectively. $\mathrm{P}_{\mathrm{FVC}}$ is scored as 0,1 , or 2 if $\mathrm{FVC}$ is $>75 \%$ predicted, $50-75 \%$ predicted, or $<50 \%$ predicted, respectively. $\mathrm{P}_{\mathrm{DLCO}}$ is scored as 0,1 , or 2 if DLCO is $>55 \%$ predicted, $36-55 \%$ predicted, or $\leq 35 \%$ predicted, respectively, or 3 if DLCO cannot be measured.

Table 1 shows a summary of the demographic data for healthy and IPF subjects, along with their full PFT results. The physiological variables of the IPF subjects indicated that their condition was mild. Their FVC and DLCO values were $85 \%$ predicted [standard deviation $(\mathrm{SD})=186 \%$ predicted $]$ and $48 \%$ predicted $(\mathrm{SD}=16 \%$ predicted), respectively. Similarly, the GAP index for the IPF subjects was $3.6(\mathrm{SD}=1.4)$, which corresponds to IPF stage I, with a predicted 3-year mortality rate of $16 \%$

Table 1 Demographic characteristics of study subjects

\begin{tabular}{|c|c|c|c|}
\hline Variable & Healthy & IPF & $p$-value \\
\hline Sex & 9 Women, 4 Men & 4 Women, 5 Men & \\
\hline Age (y) & $61(13)$ & $71(10)$ & 0.107 \\
\hline Height (in) & $66(3)$ & $65(4)$ & 0.950 \\
\hline Weight (lb) & $150(33)$ & $168(39)$ & 0.549 \\
\hline GAP index ${ }^{a}$ & $1.3(1.0)$ & $3.6(1.4)$ & 0.003 \\
\hline FVC $(\% \text { predicted })^{b}$ & $105(14)$ & $85(16)$ & 0.003 \\
\hline DLCO (\% predicted $)^{b}$ & $83(12)$ & $48(16)$ & $<0.001$ \\
\hline $\mathrm{FEV}_{1}(\% \text { predicted })^{\mathrm{b}}$ & $102(13)$ & $90(21)$ & 0.231 \\
\hline $\mathrm{FEV}_{1} / \mathrm{FVC}^{\mathrm{c}}$ & $0.75(0.05)$ & $0.81(0.05)$ & 0.010 \\
\hline TLC (\% predicted $)^{b}$ & $109(18)$ & $72(17)$ & $<0.001$ \\
\hline
\end{tabular}

Mean (standard deviation) values of demographic variables and pulmonary function test results for the healthy $(N=13)$ and IPF $(N=9)$ groups. We used the $t$-test to evaluate the statistical significance of differences in the mean values between the two groups and made the family-wise Bonferroni correction to the $p$-values. Abbreviations: DLCO diffusing capacity of carbon monoxide, $F E V$, forced expiratory volume in one second, $F V C$ forced vital capacity, GAP gender-age-physiology, IPF idiopathic pulmonary fibrosis, $T L C$ total lung capacity

${ }^{\text {a }}$ See Methods Section

${ }^{\mathrm{b}}$ Entries for FVC, DLCO, FEV 1 , and TLC are expressed as a percentage of the normal reference value (\% predicted)

${ }^{\mathrm{C}} \mathrm{FEV}_{1} / \mathrm{FVC}$ denotes the ratio of the raw volumetric measurements (in liters) of $\mathrm{FEV}_{1}$ and FVC
[20]. The TLC for the IPF subjects was $72 \%$ predicted $\left(\mathrm{SD}=17 \%\right.$ predicted). However, the $\mathrm{FEV}_{1}$ for the IPF subjects was $90 \%$ predicted ( $\mathrm{SD}=21 \%$ predicted). The $\mathrm{FEV}_{1} / \mathrm{FVC}$ for the IPF subjects was $0.81(\mathrm{SD}=0.05)$. Although most healthy subjects had normal HRCT findings and lung function, some subjects showed mild deviation from the normal range. One subject (H12) showed normal HRCT findings but a $\mathrm{FEV}_{1}$ of $79 \%$ predicted. Four subjects (H5, H6, H7, and H11) showed normal pulmonary function (e.g., $\mathrm{FEV}_{1}=84 \sim 129 \%$ predicted) but mild subpleural reticulation in the upper section (visual score $<10$ ).

\section{HRCT imaging and radiological assessment}

To compare volumetric changes in the lungs between expanded and contracted states, we collected thin-sliced $(<1 \mathrm{~mm})$ HRCT images at two breath-holding conditions, one acquired after full inhalation and another after full exhalation. To optimize imaging resolution, we followed a previously reported protocol $[26,27]$ using a SOMATOM Definition Flash scanner (Siemens, Erlangen, Germany).

For quantitative assessment, thoracic radiologists (Drs. Altes and Flors, each with more than 14 years of experience) blinded to the patient's clinical information visually scored HRCT images for the presence of subpleural reticulation, traction bronchiectasis and bronchiolectasis, ground-glass opacities, honeycombing, and emphysema, following a previously published guideline for scoring [28]. We scored variables to the nearest $5 \%$ in three zones in each lung as follows: 1) upper zones: at or above the aortic arch, 2) middle zone: between the aortic arch and inferior pulmonary veins, and 3) lower zones: at or below the inferior pulmonary veins. Table 2 shows the visual scores averaged across subjects for the healthy and IPF groups. We provide the HRCT images for each subject in the Additional file 1: Figures S1 - S22).

\section{Segmentation of lung images}

For volumetric analyses, we performed lung and lobe segmentation of HRCT images. We conducted initial lung segmentation by setting a threshold range for the Hounsfield unit (HU) of each pixel in each HRCT image in the MIMICS software system (version 11, Materialise, Leuven, Belgium). For most images, a range of -1024 to - $500 \mathrm{HU}$ was sufficient to cover all lung regions [29]. To ascertain how the upper bound influenced the segmentation, we systematically adjusted the upper bound between -700 and $-300 \mathrm{HU}$ for a number of images, and found that the change in lung volume was less than $5 \%$. Furthermore, to ensure that any difference between images of the expanded lung and contracted lung for an individual subject was not confounded by differences in the upper bound used to segment the images, we used 
Table 2 Mean (standard deviation) values of radiological scores for the healthy ( $=13)$ and IPF $(N=9)$ groups

\begin{tabular}{lllll}
\hline Variable & Zone & Healthy & IPF & $p$-value \\
\hline Subpleural reticulation & Upper & $2.31(3.88)$ & $38(27)$ & $<0.001$ \\
& Middle & $0.00(0.00)$ & $51(23)$ & $<0.001$ \\
& Lower & $0.00(0.00)$ & $72(25)$ & $<0.001$ \\
Traction & Upper & $0.00(0.00)$ & $7(10)$ & 0.003 \\
& Middle & $0.00(0.00)$ & $24(20)$ & $<0.001$ \\
& Lower & $0.00(0.00)$ & $47(32)$ & $<0.001$ \\
Ground-glass opacification & Upper & $0.00(0.00)$ & $16(10)$ & $<0.001$ \\
& Middle & $0.00(0.00)$ & $30(21)$ & $<0.001$ \\
& Lower & $0.00(0.00)$ & $49(26)$ & $<0.001$ \\
Honeycombing & Upper & $0.00(0.00)$ & $3(7)$ & 0.082 \\
& Middle & $0.00(0.00)$ & $6(11)$ & 0.082 \\
& Lower & $0.00(0.00)$ & $13(14)$ & 0.001 \\
Emphysema & Upper & $0.00(0.00)$ & $2(7)$ & 0.247 \\
& Middle & $0.00(0.00)$ & $1(3)$ & 0.247 \\
& Lower & $0.00(0.00)$ & $0(0)$ & $\mathrm{N} / \mathrm{A}$
\end{tabular}

We used the t-test to evaluate the statistical significance of differences in the meanvalues between the two groups and made the family-wise Bonferroni correction to the $p$-values. Abbreviation: IPF idiopathic pulmonary fibrosis

the same threshold range to segment the images obtained during inhalation and exhalation conditions.

In most images of healthy lungs, the initial thresholdbased segmentation was sufficient. However, for some images of IPF lungs, this segmentation procedure erroneously excluded regions with severe fibrosis from the lung domain. This was due to the difficulty of distinguishing between scarred lung tissue and its surrounding structures, for which the HU values were similar. In such cases, we manually included the left-out lung regions based on the shape and anatomy of the lung.

Next, we used the lung fissures to identify the lung lobes in the segmented lung regions. Although the major fissures were clearly identifiable for all lobes, in some cases, the minor fissure, which typically forms a border between the right upper and right middle lobes, did not exist or was not easily detectable. Because in such cases we had no systematic means of segmenting these lobes, we chose to group them together as part of the right upper lobe (RU) for all images. Thus, we segmented the lung regions into four lobes: the RU, right lower (RL), left lower (LL), and left upper (LU) lobes. Two radiologists (Drs. Altes and Flors) confirmed the identification of the lung fissures and the final segmentation results.

\section{Computation of HU distribution in lung HRCT images}

We quantitatively analyzed the distributions of $\mathrm{HU}$ values of the pixels within the lung domain of the HRCT images by computing their normalized histograms (hereafter, HU histograms). We characterized each $\mathrm{HU}$ histogram by calculating its mean, median, skewness, and kurtosis of asymmetry of a distribution. Here skewness is defined as:

$$
\text { skewness }=\frac{\overline{(x-\bar{x})^{3}}}{\sigma_{x}^{3}}
$$

where $\sigma_{x}$ denotes the standard deviation of $x$. Kurtosis indicates the extent to which a distribution contains extreme outliers, and is defined as:

$$
\text { kurtosis }=\frac{\overline{(x-\bar{x})^{4}}}{\sigma_{x}^{4}}
$$

Here, $x$ denotes the HU value of a pixel and $\bar{x}$ represents its mean across all pixels in the lung domain [30].

\section{Computation of anatomical and mechanical characteristics of lung lobes}

We quantified the anatomical and mechanical characteristics of the lung lobes using the following quantities:

Lobar volume $(V)$ : the volume of a lung lobe normalized by the volume of the whole lung.

Lobar flow $(Q)$ : the difference in the volume of a lobe between the expanded and contracted states, normalized by the difference in the lung volume between the two states. The difference in the lung volume corresponds to the total amount of air breathed. Therefore, $Q$ represents the amount of air breathed at each lobe as a fraction of this total. For each lobe $i\left(i=\mathrm{RU}, \mathrm{RL}, \mathrm{LL}\right.$, and LU), $Q_{i}$ is defined as:

$$
Q_{i}=\frac{v_{e, i}-v_{c, i}}{\sum_{i=1}^{4}\left(v_{e, i}-v_{c, i}\right)}
$$

where $v_{e, i}$ and $v_{c, i}$ denote the volumes of lobe $i$ measured in the expanded and contracted states, respectively.

Lobar strain $(S)$ : we defined the volumetric strain of each lobe $i$ ( $i=\mathrm{RU}, \mathrm{RL}, \mathrm{LL}$, and LU) as the difference in lobe volume between the expanded and contracted states, normalized by the lobe volume in the expanded state:

$$
S_{i}=\frac{v_{e, i}-v_{c, i}}{v_{e, i}}
$$

Root-mean-squared difference (RMSD) of a quantity $X$ : for each individual, this measure indicates the distance between the value of $X$ for the individual and the mean value of $X$ for the healthy group $\left(X_{i}^{h}\right)$, normalized by the standard deviation of $X$ for the healthy group $\left(\sigma_{i}^{h}\right)$. This is defined as: 


$$
\text { RMSD of } \mathrm{X}=\sum_{\mathrm{i}=1}^{4} \frac{\sqrt{\left(X_{i}-X_{i}^{h}\right)^{2}}}{4 \sigma_{i}^{h}}
$$

where lobe $i=\mathrm{RU}, \mathrm{RL}, \mathrm{LL}$, and LU.

We assessed the robustness of the RMSD of a particular quantity using the leave-one-out cross validation procedure [31], by computing the mean and standard deviation of the RMSD for 12 healthy subjects 13 times, leaving out a different healthy subject each time.

\section{Statistical analyses}

We used the t-test to assess whether there were statistically significant differences between the healthy and IPF groups. We made corrections for $p$-values using the Bonferroni method to control for the family-wise error rate [32]. For each quantity of comparison, we used the Kolmogorov-Smirnov test to confirm that the data were normally distributed [32].

\section{Results}

\section{Radiological characteristics of idiopathic pulmonary fibrosis lungs}

All IPF lungs showed HRCT features characteristic of usual interstitial pneumonia (UIP), with subpleural and basilar predominant reticulation, traction bronchiectasis, and honeycombing. Table 2 shows the group average and SD of the radiological scores. For the IPF subjects, the group-average radiological scores for subpleural reticulation, traction bronchiectasis/bronchiolectasis, ground-glass opacification, and honeycombing were highest in the lower section of the lung and lowest in the upper section. Signs of emphysema were present only in one subject, whose scores were 20 and 10 at the upper and middle sections of the lung, respectively. The healthy subjects did not show any abnormalities, except for two subjects, who each showed minimal biapical scarring (radiological score $=5$ ).

\section{Hounsfield units for healthy and idiopathic pulmonary fibrosis lungs}

To determine the radiographic differences in lung HRCT images between the healthy and IPF subjects computationally, we examined the $\mathrm{HU}$ values, which are associated with the radiological density of lung substances for the whole lung region. HU values for fibrotic lung regions (Fig. 1, red cross-marks in the right panel) were typically within a range between -200 and -600 , as observed in previous studies [33, 34].

Figure 1 shows $\mathrm{HU}$ histograms in the expanded (Fig. 1a) and contracted (Fig. 1b) lungs for the healthy and IPF subjects. Because we did not exclude pulmonary vasculature, for all subjects, the HU histogram was bimodal with peaks near HU values of -1000 (left peak) and 0 (right peak), which correspond to those for air and water, respectively [29]. However, the width and asymmetry of the left peak differed between the healthy and IPF subjects and between the expanded and contracted states. As expected for both IPF and healthy subjects, the lung density increased in the contracted state because of expiration of air from the lung. This is indicated by the reduced height and rightward shift of the left peak for both healthy and IPF subjects (Fig. 1). The mean and median $\mathrm{HU}$ values averaged across subjects for each group were higher in the contracted state (i.e., expiration) for both healthy and IPF subjects, reflecting the rightward shift of the histogram (Table 3). The skewness (Eq. 1), which quantifies the asymmetry of the histogram, was lower in the contracted state (Table 3). Similarly, kurtosis (Eq. 2), which we used to measure the extent to which the histogram included extreme outliers, was smaller in the contracted state than in the expanded state for both healthy and IPF subjects, although the difference between states was greater for the healthy subjects (Table 3 ).

Overall $\mathrm{HU}$ values for the IPF lungs were higher than those for healthy lungs in both expanded and contracted states. In both states, the left peak was broader and shifted further rightward for IPF subjects than they were for healthy subjects (Fig. 1). For all variables in both expanded and contracted states, group differences between the healthy and IPF subjects were statistically significant $(p<0.05)$ (Table 3). Moreover, the differences in the mean, median, and skewness between the IPF and healthy subjects were greater in the contracted state than in the expanded state. Specifically, average HU mean values for the IPF subjects in the expanded and contracted states were 19 and 34\% higher, respectively, than those for the healthy subjects (Table 3). Average skewness values for IPF subjects in the expanded and contracted states were 49 and $62 \%$ lower, respectively, than those for the healthy subjects (Table 3). Finally, average kurtosis values for IPF subjects in the expanded and contracted states were 60 and $40 \%$ lower, respectively, than those for the healthy subjects (Table 3).

Figure 2 shows the lobar characteristics averaged across subjects for each group, measured by the mean (Fig. 2a and b) and skewness (Fig. 2c and d) of the HU histogram in the four lobes in the expanded (left panels) and contracted (right panels) states. In both states, the HU mean, and hence lung density, was significantly larger (i.e., less negative) for the IPF subjects (Fig. 2a). Differences in the HU mean between the healthy and IPF subjects were slightly greater in the contracted state than in the expanded state (Fig. 2a): in the expanded state, HU means for the IPF subjects were roughly $20 \%$ greater than those for the healthy subjects in all lobes, whereas in the contracted state, they were 30 to $40 \%$ greater. 


\section{(a) Expanded state}
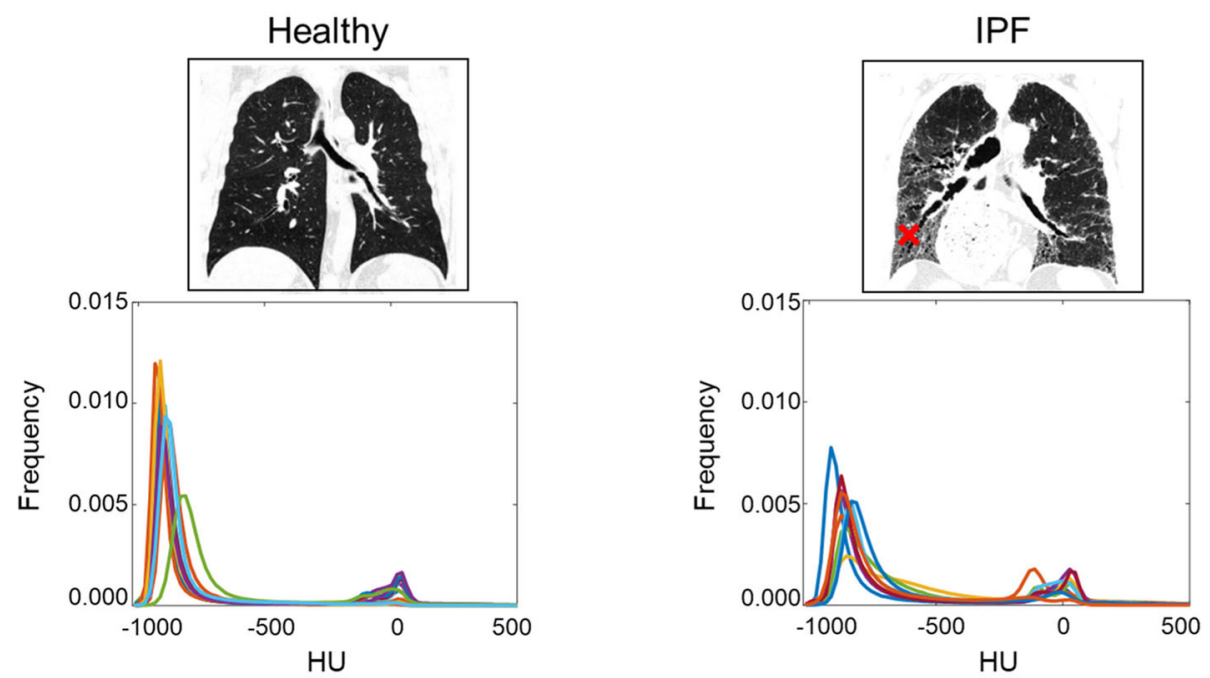

(b) Contracted state
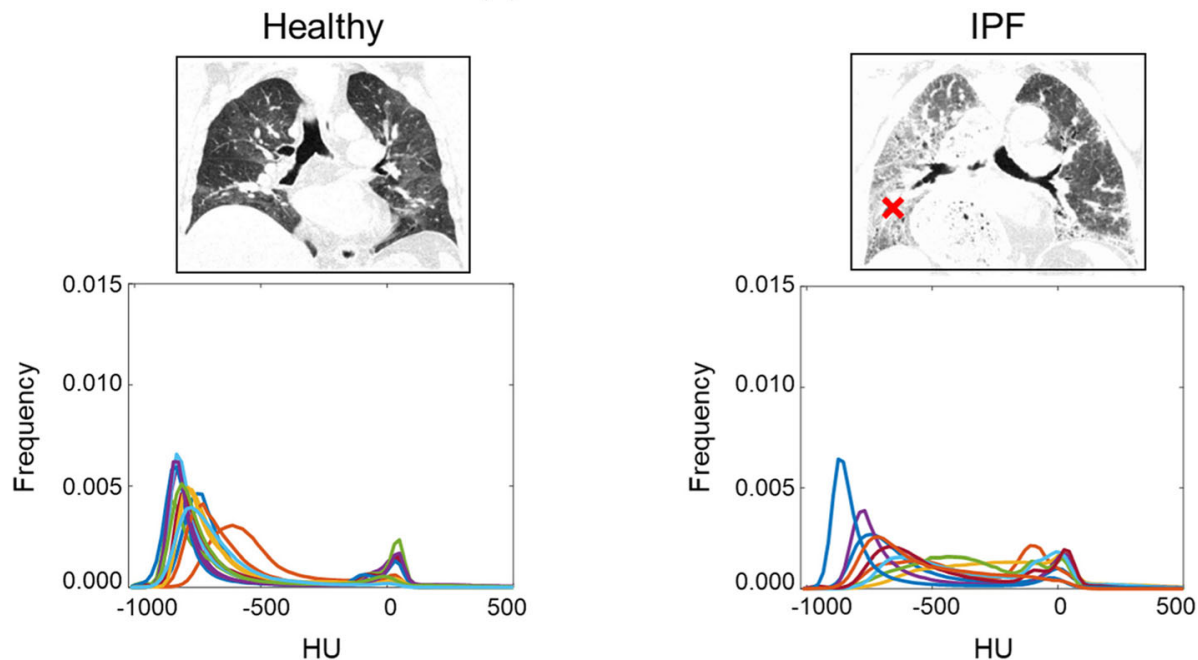

Fig. 1 High-resolution computed tomography (HRCT) images of a healthy subject and an idiopathic pulmonary fibrosis (IPF) subject and histograms of Hounsfield units (HU), normalized by the total number of occurrences, for HRCT images of healthy $(N=13$; left panel) and IPF ( $N=9$; right panel) subjects. For each subject, we acquired HRCT images at two breath-holds, one after full inspiration (Expanded; $\mathbf{a}$ ) and another after full expiration (Contracted; b). Fibrotic regions are cross-marked (red) in the HRCT images of the IPF subject. Each color in a histogram represents an individual subject

Similarly, skewness differed significantly between the healthy and IPF subjects for all lobes in both expanded and contracted states (Fig. 2c); in the expanded state, the skewness for IPF subjects was roughly $50 \%$ smaller than that for the healthy subjects, while in the contracted state, it was roughly between 56 and $66 \%$ smaller than that for the healthy group.

Figure $2 \mathrm{~b}$ and $\mathrm{d}$ show RMSD values, which quantify the distance from the average of the healthy group (Eq. 5), of the lobar mean and skewness for each individual, respectively. The RMSD of the lobar mean clearly separated the healthy and IPF subjects in the contracted state (Fig. 2b, right panel), but not in the expanded state (Fig. 2b, left panel). Similarly, the RMSD of the lobar skewness separated the healthy subjects and IPF subjects more clearly in the contracted state (Fig. 2d, right panel) than it did in the expanded state (Fig. 2d, left panel). The RMSD of the lobar skewness for one IPF subject (D9) fell within the range of the healthy group (Fig. 2d, right panel).

\section{Distribution of lobar volumes differs between healthy and idiopathic pulmonary fibrosis lungs}

Figure 3 shows the lobe volume normalized by the lung volume (lobar volume $V$, Eq. 3) in the expanded (left 
Table 3 Mean (standard deviation) values of HU for the healthy $(\mathrm{N}=13)$ and IPF $(\mathrm{N}=9)$ groups

\begin{tabular}{lllll}
\hline Variable & & Healthy & IPF & $p$-value \\
\hline Expanded state & Mean & $-739(100)$ & $-596(69)$ & 0.003 \\
& Median & $-869(48)$ & $-750(64)$ & $<0.001$ \\
& Kurtosis & $8.93(5.74)$ & $3.54(1.12)$ & 0.018 \\
& Skewness & $2.33(0.97)$ & $1.18(0.34)$ & 0.006 \\
Contracted state & Mean & $-578(60)$ & $-382(133)$ & 0.002 \\
& Median & $-690(55)$ & $-429(184)$ & 0.002 \\
& Kurtosis & $5.10(1.99)$ & $3.05(1.01)$ & 0.007 \\
& Skewness & $1.49(0.39)$ & $0.56(0.50)$ & 0.001 \\
\hline
\end{tabular}

We used the t-test to evaluate the statistical significance of differences in the mean values between the two groups and made the family-wise Bonferroni correction to the $p$-values. Abbreviations: HU Hounsfield unit, IPF idiopathic pulmonary fibrosis

panel) and contracted (right panel) states. For each lobe, the value of $V$ averaged over the subjects in each group differed between the healthy and IPF groups in both the expanded and contracted states (Fig. 3b). For the IPF group, $V$ for RU (34\% in both expanded and contracted states) was higher than that for all other lobes, which showed similar values (ranging from 21 to 23\%) in both expanded and contracted states. In contrast, for the healthy subjects, $V$ in the expanded state was greatest for RL (28\%). In the contracted state, it was greatest for RU (30\%), differing significantly $(p<0.001)$ from all other lobes where it ranged from 21 to $25 \%$.

Figure 3c shows the RMSD of $V$ for each subject. The RMSD clearly separated the healthy and IPF groups in the expanded state (Fig. 3c, left). Although the RMSD did not separate the healthy and IPF groups as well in the contracted state (Fig. 3c), it was still able to discriminate most of the subjects in the two groups.

\section{Distribution of lobar flow differs between healthy and idiopathic pulmonary fibrosis lungs}

Figure 4 shows the lobar flow $Q$ (Eq. 3), defined as the difference in lobe volume divided by difference in lung volume between the expanded and contracted states, for the healthy and IPF subjects. The $Q$ averaged across subjects for each group was 22\% (RU), 30\% (RL), 27\% (LL), and $21 \%(\mathrm{LU})$ for the healthy subjects, and $36 \%(\mathrm{RU})$, $20 \%$ (RL), 21\% (LL), and 23\% (LU) for the IPF subjects. As such, the values of $Q$ differed significantly between the healthy and IPF groups in every lobe except LU. In addition, the distribution of $Q$ across the lobes differed between the two groups. For the healthy subjects, $Q$ values for the lower lobes (30\% for RL and $27 \%$ for LL) were larger than those for the upper lobes $(22 \%$ for RU and $21 \%$ for $\mathrm{LU})$. In contrast, for the IPF subjects, $Q$ values for RU (36\%) were significantly larger $(p<0.001)$ than those for all other lobes, which were comparable (20 to $23 \%$ ).

Importantly, the $Q$ RMSD clearly separated the healthy subjects from the IPF subjects (Fig. 4c). The largest $Q$ RMSD for the healthy group (0.66 for H11) differed significantly $(p<0.001)$ from the lowest value $(1.59$ for $\mathrm{D} 4)$ for the IPF group. The $Q$ RMSD averaged across subjects was $0.46(\mathrm{SD}=0.16)$ for the healthy group and $2.56(\mathrm{SD}=$ 1.08) for the IPF group (Table 4). The $Q$ RMSD clearly grouped the healthy subjects and the IPF subjects, in agreement with their physiological results (Fig. 5).

We computed the lobar strain $S$ (Eq. 4), defined as the difference in lobe volume between the expanded and contracted states divided by the lobe volume in the expanded state (See Additional file 1: Figure S23). This metric indicates the volumetric strain of each lobe during lung contraction. $S$ did not significantly differ between the healthy and IPF subjects for any lobe (Additional file 1: Figure S23b). Not surprisingly, the $S$ RMSD could not separate the healthy subjects from the IPF subjects (Additional file 1: Figure S23c).

\section{Discussion}

In this study, we quantitatively investigated the radiological characteristics and volumetric changes of each lobe for healthy and IPF subjects. The parameters of the HU histogram profiles, such as the mean, median, skewness, and kurtosis, significantly differed between the healthy and IPF subjects in all lobes, in agreement with previous studies of individual lung lobes [22] and the whole lung [35] in IPF. Nonetheless, most of these parameters and their RMSDs did not clearly separate healthy subjects from those with IPF. The one exception was the RMSD of HU mean in the contracted state, which separated the two groups (Fig. 2b, right panel).

The difference in HU histogram profiles between the healthy and IPF subjects may indicate an increase of fibrotic areas in lungs with IPF [17]. Because fibroblasts from patients with IPF are stiffer than normal lung tissue [12], we expected that the mechanical properties of the whole lung would differ between IPF and healthy subjects. However, whether regional differences in lung function would be manifested in HRCT images was not obvious.

Notably, our study showed that quantities associated with the dynamic responses of lungs differed lobe-wise between the healthy and IPF subjects. In particular, the pattern of volume distribution among the lung lobes differed between the healthy and IPF subjects, especially in the expanded state (Fig. 3b, left panel). For the IPF subjects, RU occupied by far the largest portion of the lung volume, whereas for the healthy subjects, RL occupied the greatest proportion. It is unclear why the pattern of lobar volume distribution between the healthy 

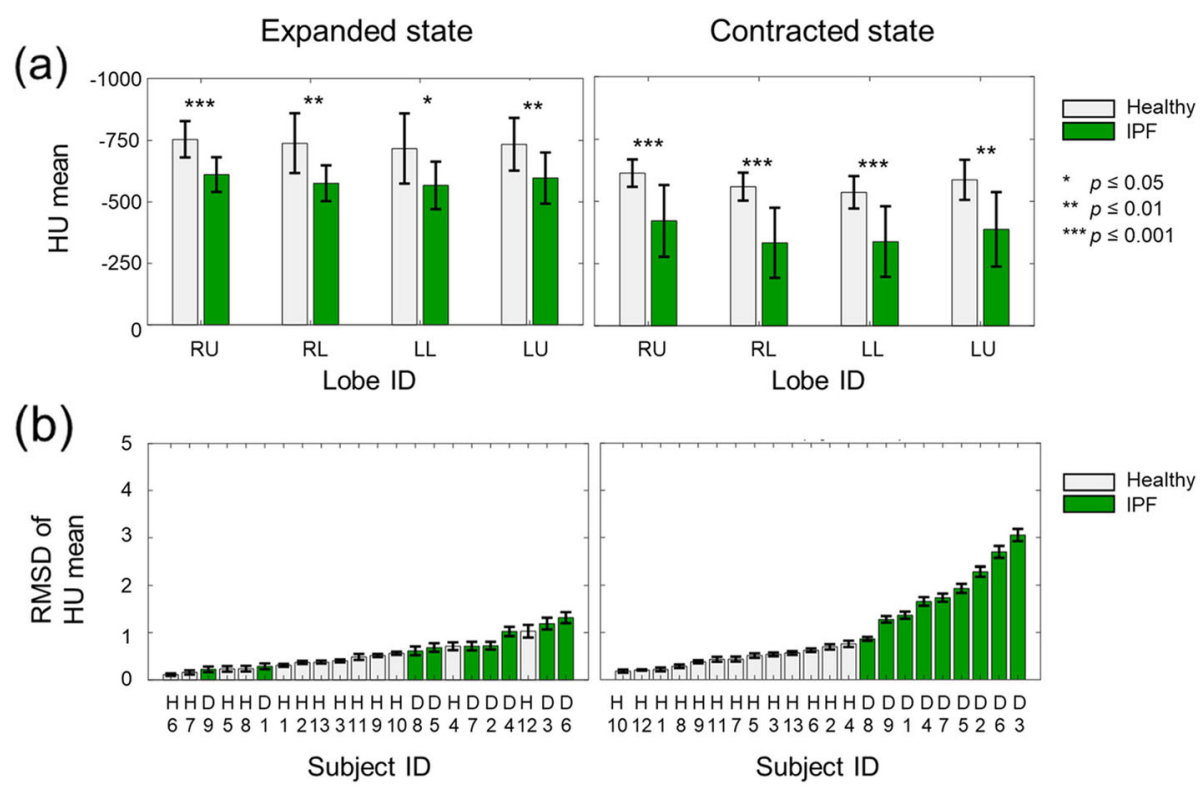

(c)
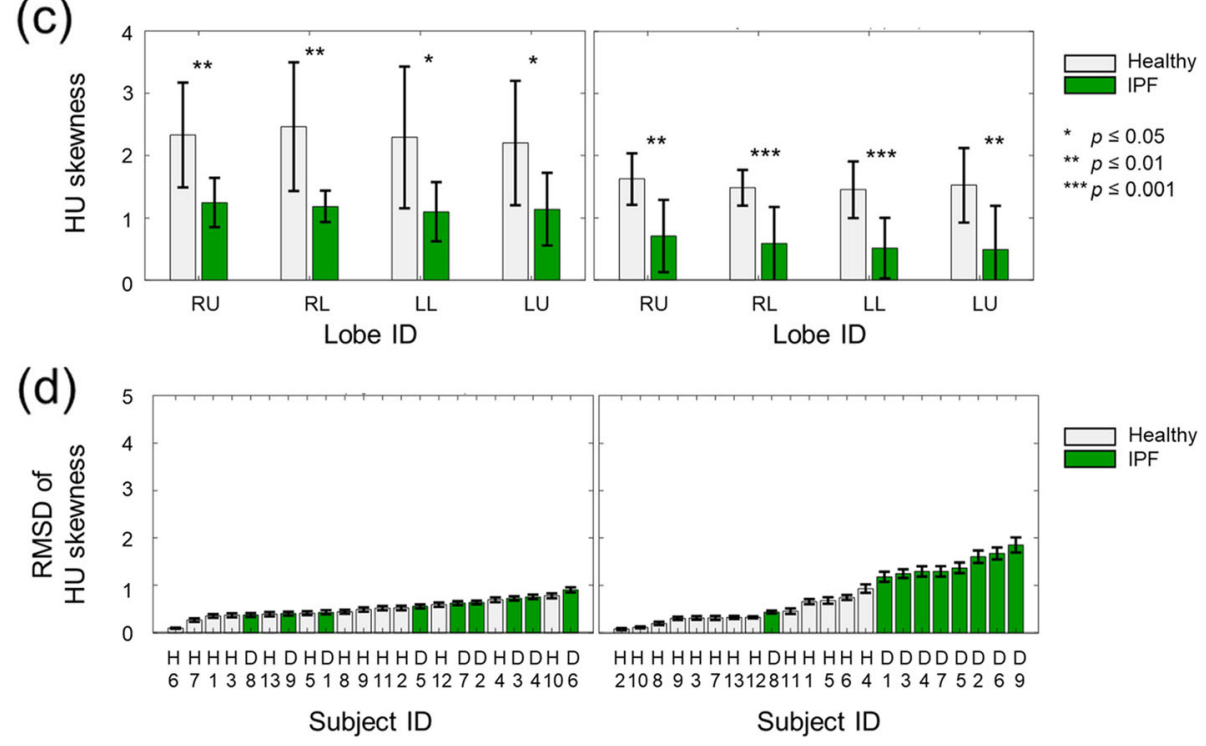

Fig. 2 Mean and skewness values of Hounsfield units (HU) of lungs in their expanded (left panel) and contracted (right panel) states for healthy $(N=13)$ and idiopathic pulmonary fibrosis (IPF) subjects $(N=9)$. We computed these values from the HU histograms shown in Fig. 1. (a) HU means averaged for the healthy and IPF groups. (b) Individual root-mean-squared difference (RMSD) values of the HU mean. (c) HU skewness values averaged for the healthy and IPF groups. (d) Individual RMSD values of HU skewness. In a and $\mathbf{c}$, the error bars denote one standard deviation and the asterisks indicate different levels of statistical significance for differences in the mean values between the two groups. In $\mathbf{b}$ and d, the error bars denote one standard deviation computed from 13 iterative leave-one-out validations (See Methods Section). RU denotes the right upper and right middle lobes combined. RL, LL, and LU denote the right lower, left lower, and left upper lobes, respectively

and IPF subjects would be more pronounced in the expanded state. Given the hysteresis of the pressurevolume loop [36], we might expect the lungs to show different dynamic characteristics during expansion and contraction. However, it is currently unknown whether fibrosis or other pathological features in IPF lungs affect expansion and contraction differently.

The presence of fibrotic tissue in the lung should increase lung stiffness in IPF subjects. However, one cannot measure lung stiffness in vivo without knowing the pressure applied to the lung during lung expansion or contraction. Nonetheless, under conditions in which the pressure in the lung is approximately uniform across the lung lobes, we can use the inverse of the lobar flow as an index of the stiffness of a lung lobe relative to the lung stiffness for each subject.

Interestingly, the distribution of lobar flows, associated with the lobar stiffness, differed between the healthy and 

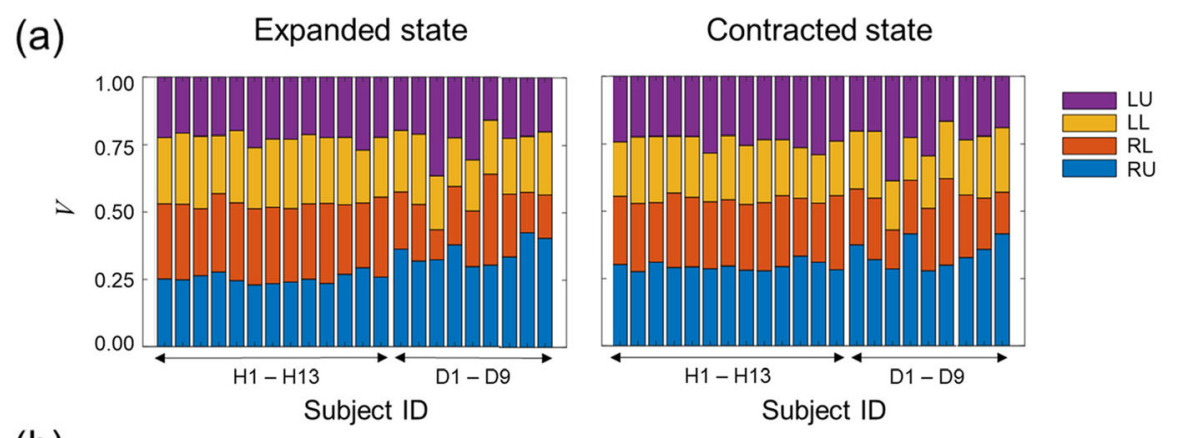

(b)
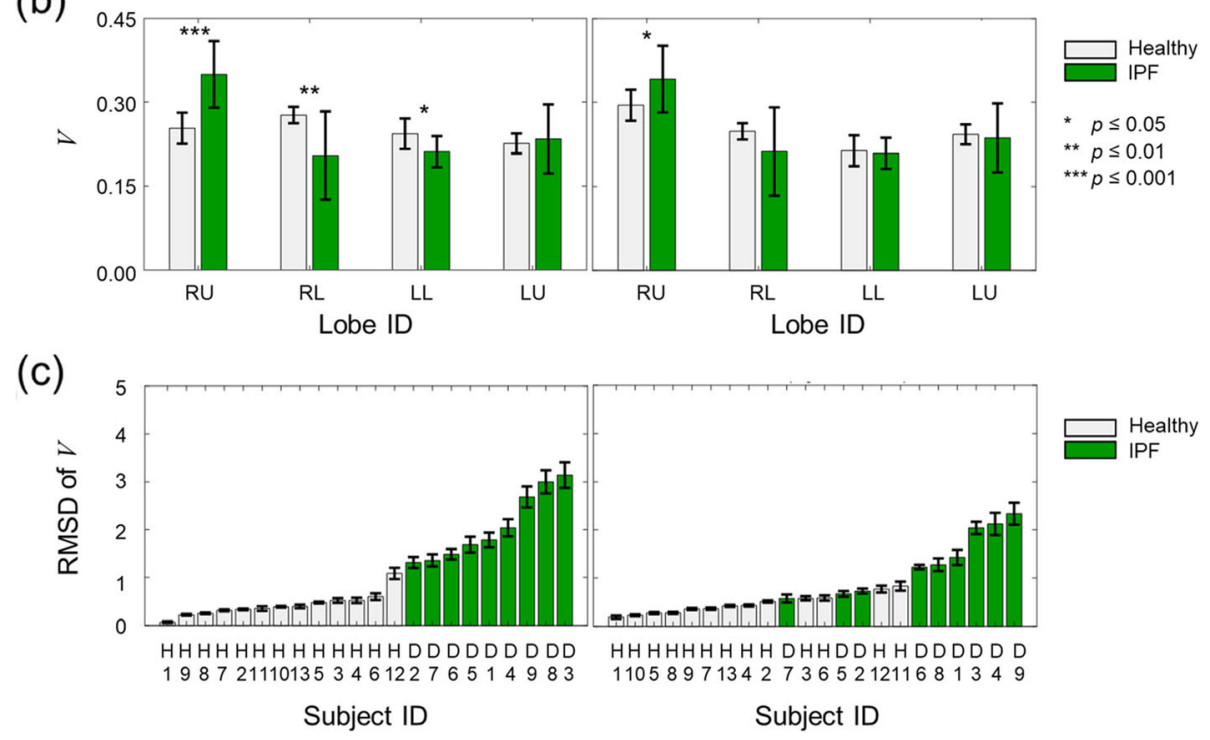

Fig. 3 The lobar volume $V$, defined as the lobe volume normalized by the lung volume, for the healthy $(N=13)$ and idiopathic pulmonary fibrosis (IPF) subjects $(N=9)$. We measured $V$ at two breath-holds, one obtained after full inspiration (expanded state) and another after full expiration (contracted state). (a) Individual $V$ values for healthy (H1-H13) and IPF subjects (D1-D9). (b) Mean values of $V$ for the healthy and IPF groups. The error bars denote one standard deviation and the asterisks indicate different levels of statistical significance for differences in the mean values between the two groups. (c) Individual root-mean-squared difference (RMSD) values of $V$ (See Eq. 5 in the Methods Section). The error bars denote one standard deviation computed from 13 iterative leave-one-out validations (See Methods Section). RU denotes the right upper and right middle lobes combined. $\mathrm{RL}, \mathrm{LL}$, and $\mathrm{LU}$ denote the right lower, left lower, and left upper lobes, respectively

IPF subjects (Fig. 4b). Specifically, the lobar flows of the lower lobes for the IPF subjects were smaller than those for the healthy subjects (Fig. 4b, RL and LL), whereas the lobar flow of the right upper lobe for the IPF subjects was higher than that for the healthy subjects (Fig. 4b, RU). This may indicate that lung stiffness for the IPF subjects in the lower lobes was higher than that in the upper lobes. In fact, visually determined radiological scores for all IPF features were highest in the lower section of the lung (Table 2), consistent with previous studies that show the greatest amount of fibrotic tissue in the lower sections $[15,18]$. Alternatively, fibrosis might not only increase the lung stiffness in the lower lobes, but also alter active mechanisms involved in lung contraction. If so, the pressure distribution across the lobes might differ between the IPF and healthy subjects, which could affect the extent of lobar contraction for the IPF subjects. Further studies to identify such mechanisms should improve our understanding of the phenomena that alter the pattern of lobar contraction in IPF subjects.

The altered distribution of lobar flows in IPF implies both global and local changes in airflow patterns. Previous studies showed that airflow patterns in individual airway branches are sensitive to the distribution of lobar flows [27, 37]. In particular, due to an increased disparity in lobar flows between lung lobes for IPF subjects (Fig. 4b), the stress on the airway branches belonging to $\mathrm{RU}$, where the flow rate is higher than other lobes, will be much higher than the stress on other airway branches. This may, in turn, induce mechanical fatigue and lead to airway damage [37].

It is noteworthy that some quantities we investigated not only differed between the healthy and IPF subjects in terms of their group averages, but also their individual (leave-one-out) RMSD values. Specifically, the RMSD of 
(a)
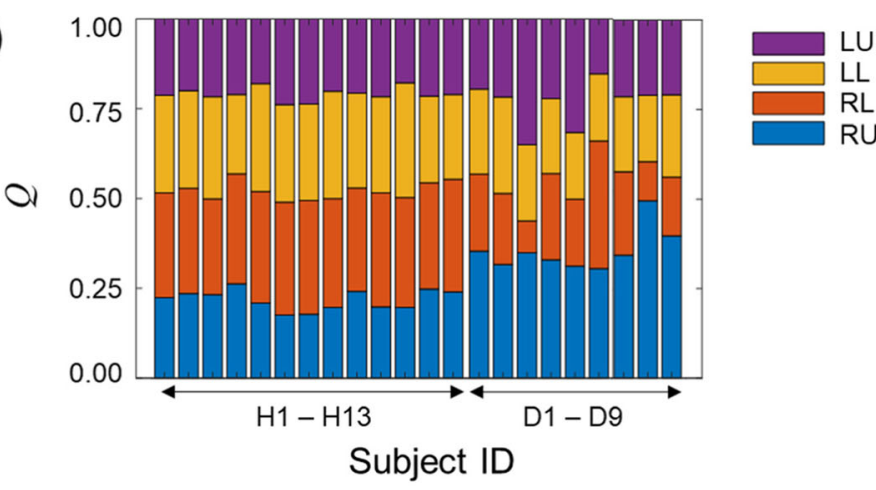

(b)

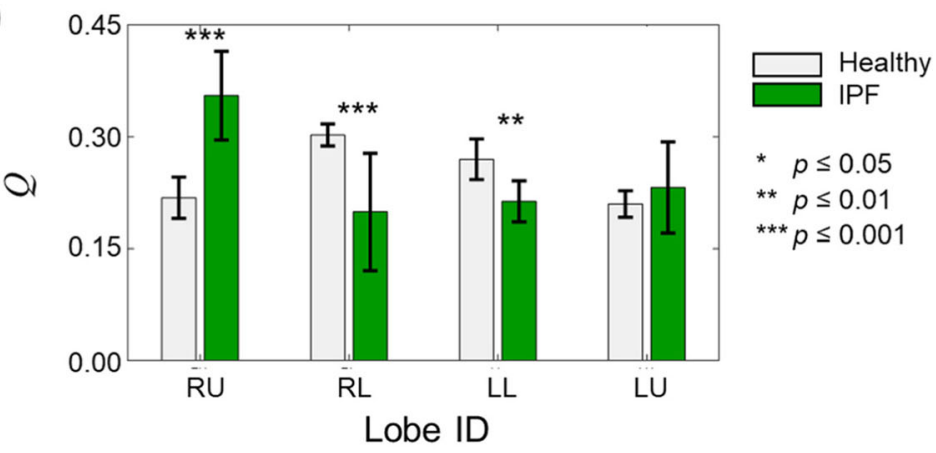

(c)

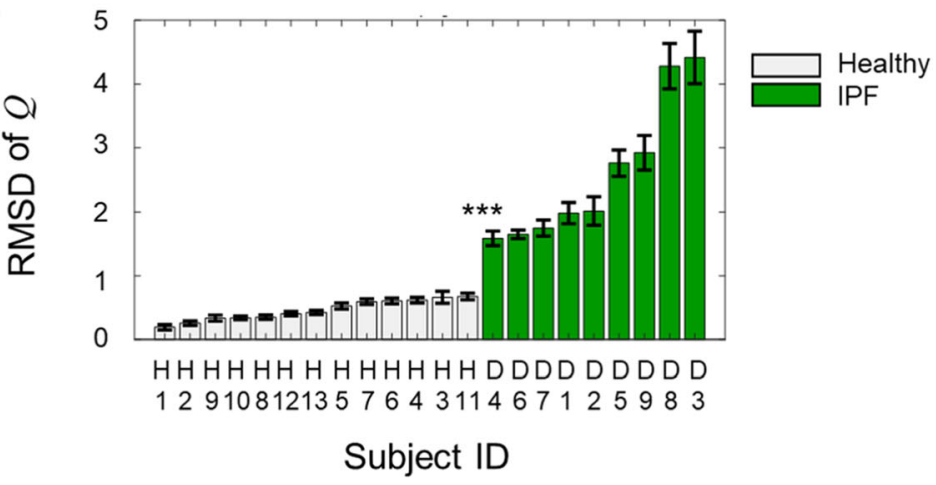

Fig. 4 The lobar flow $(Q)$ for the healthy $(N=13)$ and idiopathic pulmonary fibrosis (IPF) subjects $(N=9)$. $Q$ is defined as the difference in lobe volume normalized by the difference in lung volume between the expanded and contracted states (See Eq. 3 in the Methods Section). (a) $Q$ values of healthy (H1-H13) and IPF subjects (D1-D9). (b) Mean values of $Q$ for the healthy and IPF groups. The error bars denote one standard deviation and the asterisks indicate different levels of statistical significance for differences in the mean values between the two groups. (c) The root-mean-squared difference (RMSD) of Q for individual subjects (See Eq. 3 in the Methods Section). The error bars denote one standard deviation computed from 13 iterative leave-one-out validations (See Methods Section). RU denotes the right upper and right middle lobes combined. RL, LL, and LU denote the right lower, left lower, and left upper lobes, respectively

the HU mean in the contracted state (Fig. 2b, right panel), lobar volume in the expanded state (Fig. 3c, left panel), and lobar flow (Fig. 4c) clearly separated the healthy group from the IPF group. In particular, the RMSD of lobar flow achieved the clearest separation between the two groups, as the lower bound of the IPF group (1.59 for subject D4 in Fig. 4c) was 1.4 times the upper bound of the healthy group (0.67 for subject $\mathrm{H} 11$ in Fig. $4 \mathrm{c})$. These findings suggest that the RMSD of lobar flow could be used to differentiate IPF subjects from healthy ones.
Our study was intended as a pilot study involving a small number of subjects (13 healthy and 9 IPF subjects). Therefore, the generalizability of these results to the broader population may be limited. Hence, to test the findings identified herein, we will need to conduct a prospective investigation involving a large number of healthy and IPF subjects. In addition, it is unclear whether the differences in the HU histogram profiles and mechanical properties we observed are specific to IPF. Answering this question will require follow-up 
Table 4 Mean (standard deviation) values of volumetric variables for the healthy $(\mathrm{N}=13)$ and IPF $(\mathrm{N}=9)$ groups

\begin{tabular}{llll}
\hline Variable & Healthy & IPF & $p$-value \\
\hline Lung volume in the expanded state, $v_{e}(\mathrm{~L})$ & $5.56(0.90)$ & $4.07(0.98)$ & 0.003 \\
Lung volume in the contracted state, $v_{c}(\mathrm{~L})$ & $2.46(0.56)$ & $1.82(0.30)$ & $2.24(0.71)$ \\
Lung volume difference, $v_{e}-v_{c}(\mathrm{~L})$ & $3.10(0.91)$ & $2.15(0.23)$ & \\
Lung strain, $\left(v_{e}-v_{c}\right) / v_{e}$ & $2.20(0.46)$ & & \\
RMSD of & & $2.04(0.70)$ & 0.021 \\
Lobar volume in the expanded state & $0.43(0.24)$ & $1.36(0.66)$ & $2.56(1.08)$ \\
Lobar volume in the contracted state & $0.44(0.20)$ & 0.592 & $<0.001$ \\
Lobar flow & $0.46(0.16)$ & $0.37(0.15)$ & $<0.001$ \\
Lobar strain & $0.39(0.30)$ & 0.395 \\
\hline
\end{tabular}

We used the t-test to evaluate the statistical significance of differences in the mean values between the two groups and made the family-wise Bonferroni correction to the $p$-values. Abbreviations: IPF idiopathic pulmonary fibrosis, $L$ liter, $v_{c}$ lung volume in the contracted state, $v_{e}$ lung volume in the expanded state, RMSD root-mean-squared difference

${ }^{a}$ See Methods Section, Eq. 5

studies comparing subjects with IPF to subjects with other types of lung diseases. For example, recent reports show that comorbidity of IPF and emphysema affects disease prognosis and mortality prediction [38]. Thus, it will be of interest to compare lobar flow patterns between patients with IPF only and those with comorbidity of IPF and emphysema.

The anatomical and functional characteristics measured in our study could potentially be useful for diagnosing and characterizing IPF. Currently, it remains a challenge to accurately diagnose IPF and stage its progression given the lack of established metrics to assess disease severity. Although HRCT plays a crucial role in IPF diagnosis, an understanding of the underlying pathological changes associated with the images is lacking. Our study shows a significant increase in the stiffness of the lower lobes in IPF lungs, demonstrating that HRCT imaging can be used to probe functional (a)

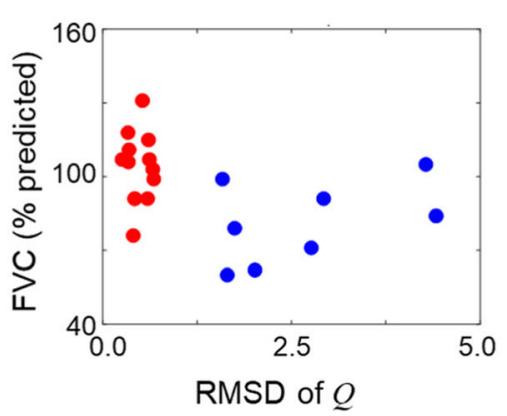

(c)

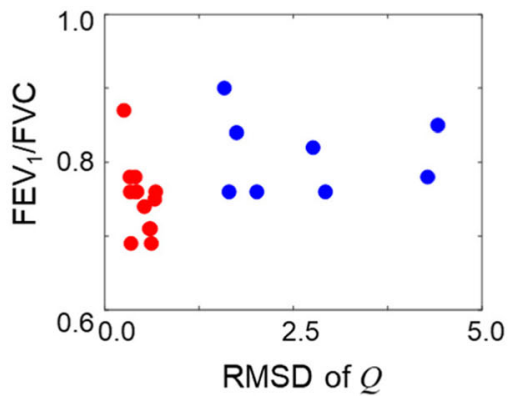

(b)

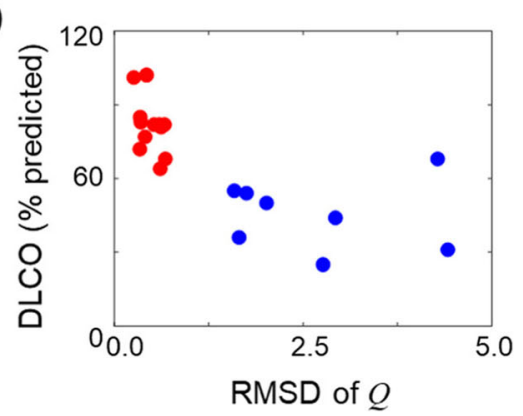

(d)

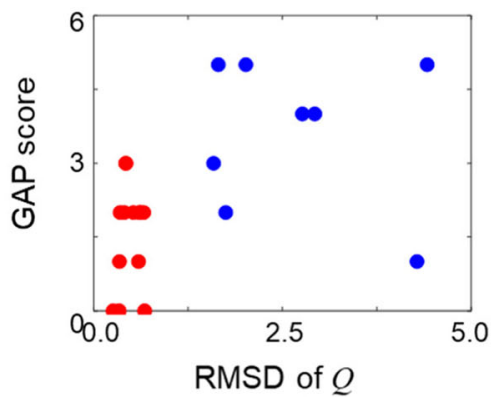

Fig. 5 The lobar flow (Q) root-mean-squared difference (RMSD) plotted as a function of the (a) forced vital capacity (FVC), (b) diffusing capacity of carbon monoxide (DLCO), (c) ratio between the forced expiratory volume in one second (FEV1) and FVC, and (d) gender-age-physiology (GAP) index (See Methods Section). The unit of \% predicted represents the value of the quantity expressed as a percentage of the normal reference value (See Methods Section). Values for healthy $(N=13)$ and idiopathic pulmonary fibrosis (IPF) subjects $(N=9)$ are shown as red and blue dots, respectively. 
impairment in different lung regions. It will be of particular interest to use HRCT imaging to investigate lung stiffness associated with regional changes in lung tissues [e.g., proliferation of fibroblasts [39]], as they are correlated with UIP patterns in thin-section CT images of IPF lungs [17]. Concurrent scoring of functional changes and staging of disease state would aid in understanding the pathogenesis and progression of IPF.

\section{Conclusions}

We performed a pilot study to investigate the structural and functional changes in the lungs of patients with IPF by quantitatively analyzing HRCT images of the lungs of healthy subjects and subjects with IPF. Hounsfield unit histograms, proportions of lobar volume, and lobar flow derived from the lung images differed significantly between healthy and IPF subjects, suggesting that both structural and functional changes in the lung occur in IPF. Interestingly, of the several statistical measures we explored for their ability to classify diseased and healthy subjects, the RMSD of lobar flow clearly separated the two subject groups. This quantity could potentially be useful in distinguishing IPF from healthy conditions.

\section{Supplementary information}

Supplementary information accompanies this paper at https://doi.org/10. 1186/s12931-019-1189-5.

Additional file 1: Figures $\mathrm{S} 1-\mathrm{S} 13$ show high-resolution computed tomography (HRCT) images and Hounsfield (HU) histograms of healthy subjects $\mathrm{H} 1$ through $\mathrm{H} 13$. Figures S14-S22 show HRCT images and HU histograms of idiopathic pulmonary fibrosis (IPF) subjects D1 through D9. Figure S23 shows the strain of the lung lobes for the healthy $(N=13)$ and IPF subjects $(\mathrm{N}=9)$.

\section{Abbreviations}

$\%$ predicted: percentage of the normal reference value; COPD: Chronic obstructive pulmonary disease; DLCO: Diffusing capacity of carbon monoxide; FEV $\mathrm{F}_{1}$ : Forced expiratory volume in one second; FVC: Forced vital capacity; HRCT: high-resolution computed tomography; HU: Hounsfield units; IPF: Idiopathic pulmonary fibrosis; PFT: Pulmonary function testing; Q: lobar flow; RMSD: Root-mean-squared difference; S: lobar strain; SD: Standard deviation; TLC: Total lung capacity; UIP: Usual interstitial pneumonia; V: lobar volume; $v_{c}$ : lung volume in the contracted state; $v_{e}$ : lung volume in the expanded state

\section{Acknowledgements}

We thank Dr. Tatsuya Oyama for editorial assistance.

\section{Disclosures}

The opinions and assertions contained herein are the private views of the authors and are not to be construed as official or as reflecting the views of the U.S. Army, DoD, or The Henry M. Jackson Foundation for Advancement of Military Medicine, Inc. This paper has been approved for public release with unlimited distribution.

\section{Authors' contributions}

$B S, T A, M M, A W$, and JR designed the study. BS conducted image segmentation, performed computational analyses of the data, interpreted the results, and drafted the manuscript. AW and JR critically contributed to interpretation of the results and manuscript preparation. TA and LF performed data collection, evaluated HRCT images, conducted visual scoring, and guided image segmentation. JC recruited study participants and collected patient data. TA provided feedback on data analyses and interpretation of the results. MM provided feedback on interpretation of the results. All authors edited the manuscript and reviewed the final version.

\section{Funding}

This research was sponsored by the U.S. Department of Defense (DoD) Health Program, managed by the Military Operational Medicine Program Area Directorate, U.S. Army Medical Research and Development Command, Fort Detrick, MD., and by the U.S. Army's Network Science Initiative.

\section{Availability of data and materials}

The datasets generated and analyzed during the current study are not publicly available due to limited consent from study participants but are available from the corresponding author on reasonable request and regulatory approval.

\section{Ethics approval and consent to participate}

This study was approved by the Human Research Protection Office at the U.S. Army Medical Research and Development Command (USAMRDC), Fort Detrick, MD, and by the Institutional Review Board at the University of Missouri, Columbia, Missouri (Review \# 233163).

\section{Consent for publication}

Participants in this study provided informed written consent for data use and publication.

\section{Competing interests}

The authors declare that they have no competing interests.

\section{Author details}

${ }^{1}$ Department of Defense Biotechnology High Performance Computing Software Applications Institute, Telemedicine and Advanced Technology Research Center, United States Army Medical Research and Development Command, FCMR-TT, 504 Scott Street, Fort Detrick, MD 21702-5012, USA.

${ }^{2}$ The Henry M. Jackson Foundation for the Advancement of Military Medicine, Inc., Bethesda 20817, MD, USA. Department of Radiology, University of Missouri, Columbia 65211, MO, USA. ${ }^{4}$ Pulmonary/Critical Care, Brooke Army Medical Center, Joint Base San Antonio, Fort Sam Houston 78234, TX, USA.

Received: 16 May 2019 Accepted: 12 September 2019 Published online: 11 October 2019

\section{References}

1. Kolb M, Collard HR. Staging of idiopathic pulmonary fibrosis: past, present and future. Eur Respir Rev. 2014;23(132):220-4.

2. Raghu G, Collard HR, Egan JJ, Martinez FJ, Behr J, Brown KK, et al. An official ats/ers/jrs/alat statement: idiopathic pulmonary fibrosis: evidence-based guidelines for diagnosis and management. Am J Respir Crit Care Med. 2011;183(6):788-824

3. Selman M, Carrillo G, Estrada A, Mejia M, Becerril C, Cisneros J, et al. Accelerated variant of idiopathic pulmonary fibrosis: clinical behavior and gene expression pattern. PLoS One. 2007;2(5):e482.

4. Ley B, Collard HR, King TE Jr. Clinical course and prediction of survival in idiopathic pulmonary fibrosis. Am J Respir Crit Care Med. 2011;183(4):431-40.

5. Selman M, King TE, Pardo A, American thoracic S, European respiratory S, American College of Chest P. Idiopathic pulmonary fibrosis: prevailing and evolving hypotheses about its pathogenesis and implications for therapy. Ann Intern Med. 2001;134(2):136-51.

6. Chilosi M, Carloni A, Rossi A, Poletti V. Premature lung aging and cellular senescence in the pathogenesis of idiopathic pulmonary fibrosis and COPD/emphysema. Transl Res. 2013;162(3):156-73.

7. Tomassetti S, Gurioli C, Ryu JH, Decker PA, Ravaglia C, Tantalocco P, et al. The impact of lung cancer on survival of idiopathic pulmonary fibrosis. Chest. 2015;147(1):157-64.

8. Liu F, Tschumperlin DJ. Micro-mechanical characterization of lung tissue using atomic force microscopy. J Vis Exp. 2011;(54):2911.

9. Alvarez D, Cardenes N, Sellares J, Bueno M, Corey C, Hanumanthu VS, et al. IPF lung fibroblasts have a senescent phenotype. Am J Physiol Lung Cell Mol Physiol. 2017;313(6):L1164-L73. 
10. Ghavami S, Yeganeh B, Zeki AA, Shojaei S, Kenyon NJ, Ott S, et al. Autophagy and the unfolded protein response promote profibrotic effects of TGF-beta1 in human lung fibroblasts. Am J Physiol Lung Cell Mol Physiol. 2018;314(3):L493-504.

11. Liu F, Lagares D, Choi KM, Stopfer L, Marinkovic A, Vrbanac V, et al. Mechanosignaling through YAP and TAZ drives fibroblast activation and fibrosis. Am J Physiol Lung Cell Mol Physiol. 2015;308(4):L344-57.

12. Jaffar J, Yang SH, Kim SY, Kim HW, Faiz A, Chrzanowski W, et al. Greater cellular stiffness in fibroblasts from patients with idiopathic pulmonary fibrosis. Am J Physiol Lung Cell Mol Physiol. 2018;315(1):L59-65.

13. Liu F, Mih JD, Shea BS, Kho AT, Sharif AS, Tager AM, et al. Feedback amplification of fibrosis through matrix stiffening and COX-2 suppression. J Cell Biol. 2010;190(4):693-706.

14. Parker MW, Rossi D, Peterson M, Smith K, Sikstrom K, White ES, et al. Fibrotic extracellular matrix activates a profibrotic positive feedback loop. J Clin Invest. 2014;124(4):1622-35

15. Raghu G, Remy-Jardin M, Myers JL, Richeldi L, Ryerson CJ, Lederer DJ, et al Diagnosis of idiopathic pulmonary fibrosis. An official ATS/ERS/JRS/ALAT clinical practice guideline. Am J Respir Crit Care Med. 2018;198(5):e44-68.

16. Tomassetti S, Piciucchi S, Tantalocco P, Dubini A, Poletti V. The multidisciplinary approach in the diagnosis of idiopathic pulmonary fibrosis: a patient case-based review. Eur Respir Rev. 2015;24(135):69-77.

17. Mai C, Verleden SE, McDonough JE, Willems S, De Wever W, Coolen J, et al. Thin-section CT features of idiopathic pulmonary fibrosis correlated with micro-CT and histologic analysis. Radiology. 2017;283(1):252-63.

18. Wells AU. The revised ATS/ERS/JRS/ALAT diagnostic criteria for idiopathic pulmonary fibrosis (IPF)-practical implications. Respir Res. 2013;14(Suppl 1):S2.

19. Bartholmai BJ, Raghunath S, Karwoski RA, Moua T, Rajagopalan S, Maldonado F, et al. Quantitative computed tomography imaging of interstitial lung diseases. J Thorac Imaging. 2013;28(5):298-307.

20. Ley B, Ryerson CJ, Vittinghoff E, Ryu JH, Tomassetti S, Lee JS, et al. A multidimensional index and staging system for idiopathic pulmonary fibrosis. Ann Intern Med. 2012;156(10):684-91.

21. Robbie H, Daccord C, Chua F, Devaraj A. Evaluating disease severity in idiopathic pulmonary fibrosis. Eur Respir Rev. 2017;26(145):170051.

22. Kim HJ, Brown MS, Chong D, Gjertson DW, Lu P, Kim HJ, et al. Comparison of the quantitative ct imaging biomarkers of idiopathic pulmonary fibrosis at baseline and early change with an interval of 7 months. Acad Radiol. 2015;22(1):70-80

23. Lynch DA, Sverzellati N, Travis WD, Brown KK, Colby TV, Galvin JR, et al. Diagnostic criteria for idiopathic pulmonary fibrosis: a Fleischner society white paper. Lancet Respir Med. 2018;6(2):138-53.

24. NHANES III reference manuals and reports. Hyattsville, MD: Data Dissemination Branch; 1996.

25. Quanjer PH, Tammeling GJ, Cotes JE, Pedersen OF, Peslin R, Yernault JC. Lung volumes and forced ventilatory flows. Eur Respir J. 1993;6(Suppl 16):5-40.

26. Yin Y, Choi J, Hoffman EA, Tawhai MH, Lin CL. Simulation of pulmonary air flow with a subject-specific boundary condition. J Biomech. 2010;43(11):2159-63.

27. Sul B, Oppito Z, Jayasekera S, Vanger B, Zeller A, Morris M, et al. Assessing airflow sensitivity to healthy and diseased lung conditions in a computational fluid dynamics model validated in vitro. J Biomech Eng. 2018;140(5):051009-14.

28. Best AC, Meng J, Lynch AM, Bozic CM, Miller D, Grunwald GK, et al. Idiopathic pulmonary fibrosis: physiologic tests, quantitative $C T$ indexes, and CT visual scores as predictors of mortality. Radiology. 2008;246(3):935-40.

29. Kazerooni EA, Gross BH. Cardiopulmonary imaging. Philadelphia: Lippincott Williams \& Wilkins; 2004.

30. Kenney JF, Keeping ES. Mathematical statistics. 2nd ed. Princeton: Van Nostrand Company; 1962.

31. Stone M. Cross-validatory choice and assessment of statistical predictions. J R Stat Soc Ser B Methodol. 1974;36(2):111-33.

32. Michael H, CJN K, Neter J, Li WI. Applied linear regression models. 5th ed. New York: McGraw-Hill; 2004

33. Ash SY, Harmouche R, Vallejo DL, Villalba JA, Ostridge K, Gunville R, et al. Densitometric and local histogram based analysis of computed tomography images in patients with idiopathic pulmonary fibrosis. Respir Res. 2017;18(1):45.

34. Lederer DJ, Enright PL, Kawut SM, Hoffman EA, Hunninghake G, van Beek EJ, et al. Cigarette smoking is associated with subclinical parenchymal lung disease: the multi-ethnic study of atherosclerosis (MESA)-lung study. Am J Respir Crit Care Med. 2009;180(5):407-14.
35. Sverzellati N, Calabro E, Chetta A, Concari G, Larici AR, Mereu M, et al. Visual score and quantitative $\mathrm{CT}$ indices in pulmonary fibrosis: relationship with physiologic impairment. Radiol Med. 2007;112(8):1160-72.

36. Suki B, Stamenovic D, Hubmayr R. Lung parenchymal mechanics. Compr Physiol. 2011;1(3):1317-51

37. Sul B, Wallqvist A, Morris MJ, Reifman J, Rakesh V. A computational study of the respiratory airflow characteristics in normal and obstructed human airways. Comput Biol Med. 2014;52:130-43.

38. Schmidt SL, Nambiar AM, Tayob N, Sundaram B, Han MK, Gross BH, et al. Pulmonary function measures predict mortality differently in IPF versus combined pulmonary fibrosis and emphysema. Eur Respir J. 2011;38(1):176-83.

39. Sgalla G, lovene B, Calvello M, Ori M, Varone F, Richeldi L. Idiopathic pulmonary fibrosis: pathogenesis and management. Respir Res. 2018;19(1):32.

\section{Publisher's Note}

Springer Nature remains neutral with regard to jurisdictional claims in published maps and institutional affiliations.
Ready to submit your research? Choose BMC and benefit from:

- fast, convenient online submission

- thorough peer review by experienced researchers in your field

- rapid publication on acceptance

- support for research data, including large and complex data types

- gold Open Access which fosters wider collaboration and increased citations

- maximum visibility for your research: over $100 \mathrm{M}$ website views per year

At BMC, research is always in progress.

Learn more biomedcentral.com/submissions 